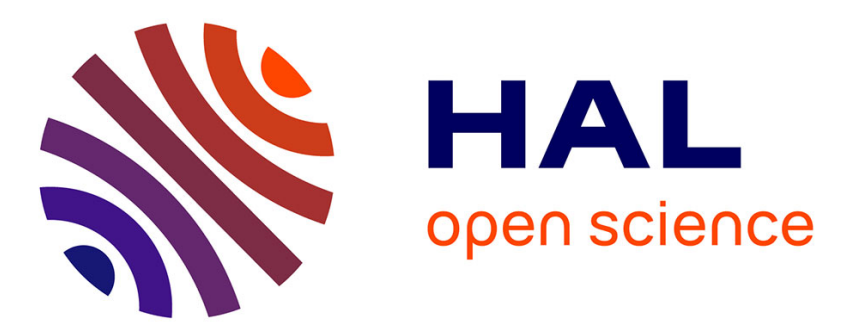

\title{
Improper choosability of graphs and maximum average degree
}

Frédéric Havet, Jean-Sébastien Sereni

\section{To cite this version:}

Frédéric Havet, Jean-Sébastien Sereni. Improper choosability of graphs and maximum average degree. RR-5164, INRIA. 2004. inria-00071425

\section{HAL Id: inria-00071425 https://hal.inria.fr/inria-00071425}

Submitted on 23 May 2006

HAL is a multi-disciplinary open access archive for the deposit and dissemination of scientific research documents, whether they are published or not. The documents may come from teaching and research institutions in France or abroad, or from public or private research centers.
L'archive ouverte pluridisciplinaire HAL, est destinée au dépôt et à la diffusion de documents scientifiques de niveau recherche, publiés ou non, émanant des établissements d'enseignement et de recherche français ou étrangers, des laboratoires publics ou privés. 
INSTITUT NATIONAL DE RECHERCHE EN INFORMATIQUE ET EN AUTOMATIQUE

\title{
Improper choosability of graphs and maximum average degree
}

\author{
Frédéric Havet — Jean-Sébastien Sereni
}

$\mathbf{N}^{\circ} 5164$

Mars 2004

THÈME 1 



\title{
Improper choosability of graphs and maximum average degree
}

\author{
Frédéric Havet*, Jean-Sébastien Sereni ${ }^{\dagger}$ \\ Thème 1 - Réseaux et systèmes \\ Projet Mascotte \\ Rapport de recherche $n^{\circ} 5164$ - Mars $2004-19$ pages
}

\begin{abstract}
Improper choosability of planar graphs has been widely studied. In particular, Škrekovski investigated the smallest integer $g_{k}$ such that every planar graph of girth at least $g_{k}$ is $k$-improper 2-choosable. He proved [8] that $6 \leq g_{1} \leq 9 ; 5 \leq g_{2} \leq 7 ; 5 \leq g_{3} \leq 6$ and $\forall k \geq 4, g_{k}=5$. In this paper, we study the greatest real $M(k, l)$ such that every graph of maximum average degree less than $M(k, l)$ is $k$-improper $l$-choosable. We prove that for $l \geq 2$ then $M(k, l) \geq l+\frac{l k}{l+k}$. As a corollary, we deduce that $g_{1} \leq 8$ and $g_{2} \leq 6$. We also provide an upper bound for $M(k, l)$. This implies that for any fixed $l, M(k, l) \underset{k \rightarrow \infty}{\longrightarrow} 2 l$.
\end{abstract}

Key-words: improper colouring, choosability, maximum average degree, planar graph, girth

This work was partially supported by the european project CRESCCO

*havet@sophia.inria.fr

† sereni@sophia.inria.fr 


\section{Choisissabilité impropre et degré maximum moyen}

Résumé : La choisissabilité impropre des graphes planaires a été beaucoup étudiée. En particulier, Škrekovski a étudié le plus petit entier $g_{k}$ tel que tout graphe planaire de maille au moins $g_{k}$ soit $k$-impropre 2-choisissable. Il a prouvé [8] les résultats suivants : $6 \leq g_{1} \leq$ $9 ; 5 \leq g_{2} \leq 7 ; 5 \leq g_{3} \leq 6$ et $\forall k \geq 4, g_{k}=5$. Dans cet article, nous introduisons le plus grand réel $M(k, l)$ tel que tout graphe de degré moyen maximum strictement inférieur à $M(k, l)$ soit $k$-impropre $l$-choisissable. Nous prouvons que $\forall l \geq 2, M(k, l)+\frac{l k}{l+k}$. Nous déduisons en corollaire que $g_{1} \leq 8$ et $g_{2} \leq 6$. Nous donnons également une borne supérieure pour $M(k, l)$ dont on déduit que, pour tout $l$ fixé, $M(k, l) \underset{k \rightarrow+\infty}{\longrightarrow} 2 l$.

Mots-clés : coloration impropre, choisissabilité, degré moyen maximum, graphe planaire, maille 


\section{Introduction.}

Let $G$ be a graph. We note $V(G)$ its vertex set and $E(G)$ its edge set.

A colouring is an application from the vertex set into a set of colours $S$. If $|S|=l$ we call it $l$-colouring. Let $c$ be a colouring of $G$. The improperty of a vertex $v$ in $G$ under $c$, denoted by $i_{G}^{c}(v)$, is the number of neighbours $u$ of $v$ in $G$ such that $c(u)=c(v)$. The improperty of $c$ in $G$ is $i m_{G}(c)=\max \left\{i m_{G}^{c}(v) \mid v \in V(G)\right\}$. A colouring is $k$-improper if its improperty is at most $k$ and a graph is $k$-improper $l$-colourable if it admits a $k$-improper $l$ colouring. The k-improper chromatic number of $G$, denoted by $c_{k}(G)$, is the smallest integer $l$ such that $G$ is $k$-improper $l$-colourable. Note that 0 -improper colouring is the usual notion of proper colouring, so the 0 -improper chromatic number is exactly the chromatic number usually denoted $\chi(G)$.

One can analogously generalize the notion of choosability. A list-assignment of a graph $G$ is an application $L$ which assigns to each vertex $v \in V(G)$ a prescribed list of colours $L(v)$. $L$ is an $l$-list-assignment provided each list is of size at least $l . G$ is $k$-improper $L$-colourable if there exists a $k$-improper colouring $c$ of $G$ such that $\forall v \in V(G), v \in L(v)$. In this case, $c$ is a $k$-improper $L$-colouring of $G$. $G$ is $k$-improper $l$-choosable if it is $k$-improper $L$-colourable for every $l$-list-assignment $L$.

Colourings of planar graphs have been widely studied. In particular $p_{k}$ and $p_{k}^{*}$, the smallest integers $l$ such that every planar graph is $k$-improper $l$-colourable and $k$-improper $l$-choosable respectively, are known for almost all $k$. Indeed Thomassen showed in [9] that every planar graph is 5-choosable and there are planar graphs which are not 4-choosable [12] so $p_{0}^{*}=5$. Every planar graph is 4-colourable $[1,2]$ and there are graphs which are not 1-improper 3-colourable, so $p_{0}=p_{1}=4$. But we do not know the exact value of $p_{1}^{*}$ which is either 4 or 5 . However, it is conjectured that it is 4 :

Conjecture 1 (Eaton and Hull [3], Škrekovski [6]) Every planar graph is 1-improper 4-choosable.

As shown independently by Eaton and Hull [3] and Škrekovski [6], every planar graph is 2 -improper 3-choosable and for every $k$, there are planar graphs which are not $k$-improper 2-colourable. Hence $p_{k}=p_{k}^{*}=3$ for any $k \geq 2$.

Moreover improper colourings of planar graphs have also been studied under some girth restrictions. The girth of graph is the smallest length of a cycle. The well-known theorem of Grötzsch $[4,11]$ states that every planar graph of girth at least 4 is 3 -colourable. Voigt [13] showed a planar graph of girth 4 which is not 3-choosable and Thomassen [10] proved that every planar graph of girth at least 5 is 3-choosable. In [7], Škrekovski showed that every planar graph of girth at least 4 is 1 -improper 3 -choosable. In [8], Škrekovski investigated $k$ improper 2-choosability of planar graphs in relation with their girth. Denoting by $g_{k}$ be the smallest integer such that every planar graph of girth at least $g_{k}$ is $k$-improper 2-choosable, he proved that $6 \leq g_{1} \leq 9,5 \leq g_{2} \leq 7,5 \leq g_{3} \leq 6$ and $\forall k \geq 4, g_{k}=5$. Hence the only unknown values are $g_{1}, g_{2}$ and $g_{3}$.

In this paper, we study the $k$-improper $l$-choosability of graphs in relation with their maximum average degree.

$\mathrm{RR} \mathrm{n}^{\circ} 5164$ 
Definition 1 The maximal average degree of a graph $G$ is:

$$
\operatorname{Mad}(G):=\max \left\{\frac{\sum_{v \in V(H)} d_{H}(v)}{|V(H)|}, H \text { subgraph of } G\right\} .
$$

The girth and the maximum average degree of a planar graph are related to each other:

Theorem 1 Let $G$ be a planar graph of girth $g$.

$$
\operatorname{Mad}(G)<\frac{2 g}{g-2} .
$$

Proof. We recall the Euler's formula for a planar graph $H:|V(H)|-|E(H)|+|F(H)|=2$ with $|F(H)|$ the number of faces of $H$. Note that every subgraph $H$ of $G$ has girth at least $g$, so $g|F(H)| \leq 2|E(H)|$. Thus $2 g-g|V(H)|+g|E(H)|=g|F(H)| \leq 2|E(H)|$. Hence $\frac{2|E(H)|}{|V(H)|} \leq \frac{2 g}{g-2}-\frac{4 g}{(g-2)|V(H)|}<\frac{2 g}{g-2}$ for every subgraph $H$ of $G$.

Let $M(k, l)$ be the greatest real such that every graph of maximum average degree less than $M(k, l)$ is $k$-improper $l$-choosable. Obviously, $M\left(k_{1}, l\right) \leq M\left(k_{2}, l\right)$ if $k_{1} \leq k_{2}$.

We have that $M(k, 1)=\frac{2 k+2}{k+2}$ since a graph is $k$-improper 1-choosable if, and only if, it has maximum degree at most $k$.

In order to introduce our method which uses some discharging process, we first present it in Section 2 for improper 2-choosability: we prove that for every $k \geq 0$,

$$
4-\frac{4}{k+2} \leq M(k, 2) \leq 4-\frac{2 k+4}{k^{2}+2 k+2} .
$$

As a corollary, we obtain the following upper bounds for $g_{k}$ which are better than Škrekovski's ones: $g_{1} \leq 8, g_{2} \leq 6, g_{3} \leq 6$ and $\forall k \geq 4, g_{k}=5$.

In Section 3 we extend the lower bound of Section 2 to any value of $l$ : we prove that for every $l \geq 2$ and $k \geq 0$,

$$
l+\frac{l k}{l+k} \leq M(k, l)
$$

Last, we provide for any value of $l$ and $k$ a graph which is not $k$-improper $l$-choosable, and we deduce that $M(k, l) \underset{k \rightarrow \infty}{\longrightarrow} 2 l$.

\section{Improper 2-choosability}

\subsection{Lower bound for $M(k, 2)$}

In this subsection, we shall prove the following theorem:

Theorem 2 For all $k \geq 0$, all graphs of maximum average degree less than $\frac{4 k+4}{k+2}$ are $k$ improper 2-choosable. 
Note that if $k=0$ the result holds trivially. Indeed a graph with maximum average degree less than 2 contains no cycle and so is a forest. Hence it is 2-choosable. Furthermore $M(0,2) \leq 2$ since an odd cycle is not 2-colourable, so $M(0,2)=2$.

For bigger value of $k$, we will need the following preliminary definitions and results:

Definition 2 If $v \in V(G)$ then $d_{G}(v)$ denotes the degree of $v$ in the graph $G$. For all positive integer $d$, a vertex of degree equals to (resp. at most, resp. at least) $d$ is called a $d$-vertex (resp. $(\leq d)$-vertex, resp. $(\geq d)$-vertex). For $S \subseteq V(G)$ (resp. $E \subseteq E(G)$ ) we denote by $G-S$ (resp. $G-E$ ) the induced subgraph of $G$ obtained by removing the vertices (resp. edges) of $S$ (resp. $E$ ) from $V(G)$ (resp. $E(G)$ ). If $S=\{v\}$ and $E=\{u v\}$, we shall note $G-v=G-S$ and $G-u v=G-E$. The union (resp. intersection) of the graphs $G_{1}$ and $G_{2}$ is the graph $G=G_{1} \cup G_{2}$ (resp. $G=G_{1} \cap G_{2}$ ) such that $V(G)=V\left(G_{1}\right) \cup V\left(G_{2}\right)$ (resp. $V(G)=V\left(G_{1}\right) \cap V\left(G_{2}\right)$ ) and $E(G)=E\left(G_{1}\right) \cup E\left(G_{2}\right)$ (resp. $E(G)=E\left(G_{1}\right) \cap E\left(G_{2}\right)$ ).

A graph is said to be $(k, 2)$-minimal if it is not $k$-improper 2-choosable but each of its proper subgraphs is.

Lemma 1 (Škrekovski [8]) Let $k \geq 1$ and let $G$ be a $(k, 2)$-minimal graph. Then

(i) $\delta \geq 2$.

(ii) Two $(\leq k+1)$-vertices are not adjacent.

Definition 3 Let $D$ be a digraph. The outdegree (resp. indegree) of a vertex $u$ in $D$ is denoted by $d_{D}^{+}(u)$ (resp. $d_{D}^{-}(u)$ ). The degree of $u$ is $d_{D}(u)=d_{D}^{-}(u)+d_{D}^{+}(u)$; it is the degree of $u$ in the underlying undirected graph.

If $u$ and $v$ are two of its vertices, a $(u, v)$-dipath is a directed path from $u$ to $v$.

An arborescence is an oriented tree in which every path is directed from a vertex called the root. Note that in an arborescence every vertex except the root has indegree 1 . The leaves of the arborescence are the vertices of outdegree 0 . A vertex which is neither a leaf nor the root is an internal vertex. A quasi-arborescence is a directed graph obtained from an arborescence by identifying some leaves.

Let $u$ be a vertex of a digraph $D$. The outsection of $u$ in $D$, denoted $A_{D}^{+}(u)$, is the set of vertices $v$ such that there is a $(u, v)$-dipath in $D$.

Let $G$ be a $(k, 2)$-minimal graph. We partially orient $G$ using the following process:

1. Orient each edge $u v$ where $v$ is a 2-vertex from $u$ to $v$.

2. If $k \geq 3$, orient each edge $u v$ where $v$ is a 3 -vertex from $u$ to $v$.

3. While there is an unoriented edge $u v$ where $v$ an $i$-vertex with $2+k \leq i<\frac{3 k}{2}+2$ and outdegree $i-1$, we orient it from $u$ to $v$.

The digraph $D$ induced by the oriented edges is called a discharging digraph of $G$.

The following proposition, whose proof is left to the reader, follows immediately from the definition of a discharging digraph.

$\mathrm{RR} \mathrm{n}^{\circ} 5164$ 
Proposition 1 Let $D$ be a discharging digraph of a $(k, 2)$-minimal graph.

- D has no 2-circuit since two $(\leq k+1)$-vertices are not adjacent by Lemma 1 (ii). So it has no circuit at all.

- If $k \leq 2$, only vertices of degree 2 or $k+2$ have indegree more than zero. If $k \leq 3$, only vertices of degree 2,3 or $k+2$ have indegree more than zero.

- Every 2-vertex has indegree exactly 2 in $D$ and if $k \geq 3$, every 3-vertex has indegree exactly 3.

- For every vertex $u, A_{D}^{+}(u)$ is a quasi-arborescence whose leaves have degree 2 (resp. 2 or 3 ) in $G$ if $k \leq 2$ (resp. $k \geq 3$ ). In particular, the indegree of the leaves in $A_{D}^{+}(u)$ is at most 2 (resp. 3).

Definition 4 A quasi-arborescence is a $(k, 2)$-quasi-arborescence if and only if:

- Every vertex has outdegree at most $\max \{2,2 k-1\}$.

- Every leaf has indegree at most $\min \{k, 3\}$.

Lemma 2 Let $k \geq 2$. Let $Q$ be a $(k, 2)$-quasi-arborescence rooted at $u$ and $L$ a 2-listassignment of $Q$. Then any L-colouring of the leaves can be extended in a $k$-improper $L$-colouring of $D$ such that $u$ has improperty at most $k-1$.

Proof. By induction on the number of vertices of $Q$, the result being trivially true if $|V(Q)|=1$.

Suppose now that $|V(Q)|>1$ and the result holds for smaller $k$-quasi-arborescences. Let $v_{1}, \ldots, v_{s}$ be the outneighbours of $u$ in $Q$. Note that $Q-u$ is the union of $s(k, 2)$ quasi-arborescences $Q_{i}, 1 \leq i \leq s$ rooted at $v_{i}$ that are disjoint except possibly on their leaves.

Let $c$ be an $L$-colouring of the leaves of $Q$. Then by induction it can be extended in a $k$-improper $L$-colouring of each of the $Q_{i}$ so that $i m\left(v_{i}\right) \leq k-1$. Since a leaf of $Q$ has indegree at most $\min \{k, 3\}$ and $i m_{Q}(x)=i m_{Q_{i}}(x)$ for every vertex of $Q_{i}$ which is not a leaf, then the union of these colourings is a $k$-improper $L$-colouring of $Q$ such that $i m\left(v_{i}\right) \leq k-1$.

Now, one of the two colours of $L(u)$, say $\alpha$, is assigned to at most $k-1$ neighbours of $u$ since $s \leq 2 k-1$. Thus setting $c(u)=\alpha$, we obtain the desired colouring.

Obviously, the above result cannot be extended for $k=1$ because it is hopeless to extend every $L$-colouring of the leaves in a colouring such that the root has improperty 0 . However, one can prove the following weaker result:

Lemma 3 Let $Q$ be a (1,2)-quasi-arborescence rooted at $u, L$ a 2-list-assignment of $Q$ with $L(u)=\{\alpha, \beta\}$ and $c$ an $L$-colouring of $S$ the set of leaves of $Q$ with indegree 1 . One the following holds: 
(i) c may be extended in a 1-improper $L$-colouring of $Q$ such that $i m(u)=0$;

(ii) $c$ may be extended in two different 1-improper $L$-colourings of $Q$, one such that $c(u)=$ $\alpha$ and one such that $c(u)=\beta$.

Proof. We proceed by induction on the number of vertices of $Q$. Let $v_{1}$ an $v_{2}$ be two outneighbours of $u$ in $Q . Q-u$ is the union of two (1,2)-quasi-arborescences $Q_{1}$ and $Q_{2}$, rooted at $v_{1}$ and $v_{2}$ respectively, that are disjoint except possibly on their leaves. Let $S^{\prime}$ be the set of leaves in $Q_{1} \cap Q_{2}$ and $L(u)=\{\alpha, \beta\}$. We $L$-colour the leaves of $Q_{i}$ that have indegree 1 in $Q_{i}$. By induction, each of the $Q_{i}$ satisfies $(i)$ or $(i i)$.

If at least one of the $Q_{i}$ satisfies (ii), then one can extend $c$ to $Q_{1} \cup Q_{2}$ such that $\left\{c\left(v_{1}\right), c\left(v_{2}\right)\right\} \neq L(u)$, say $\alpha \notin\left\{c\left(v_{1}\right), c\left(v_{2}\right)\right\}$. Moreover for any vertex $x$ not in $V\left(Q_{i}\right) \backslash S^{\prime}$, $i m_{Q}(x)=i m_{Q_{i}}(x) \leq 1$. If a vertex $s^{\prime} \in S^{\prime}$ has improperty 2 then its two neighbours are coloured the same. So recolouring $s^{\prime}$ with the colour of $L\left(s^{\prime}\right) \backslash\left\{c\left(s^{\prime}\right)\right\}$, we get a 1-improper $L$-colouring of $Q_{1} \cup Q_{2}$. Hence setting $c(u)=\alpha$, we get a 1 -improper $L$-colouring of $Q$ such that $i m(u)=0$. Thus $Q$ satisfies (i).

Suppose now $Q_{1}$ and $Q_{2}$ both satisfy (i). Then, possibly with recolouring of vertices of $S^{\prime}$ as before, one can extend $c$ into a 1-improper $L$-colouring of $Q_{1} \cup Q_{2}$ such that $i m\left(v_{1}\right)=i m\left(v_{2}\right)=0$. If $\left\{c\left(v_{1}\right), c\left(v_{2}\right)\right\} \neq L(u)$, say $\alpha \notin\left\{c\left(v_{1}\right), c\left(v_{2}\right)\right\}$ then setting $c(v)=\alpha$, we get a 1-improper $L$-colouring of $Q$ such that $i m(u)=0$. Thus $Q$ satisfies (i). If not then assigning to $u$ the colours $\alpha$ and $\beta$, we get the two 1-improper $L$-colourings of $Q$ satisfying (ii).

Lemma 4 Let $k \geq 3$. Let $D$ be a discharging digraph of a $(k, 2)$-minimal graph $G$.

(i) Every $i$-vertex with $4 \leq i \leq k+1$ has outdegree zero.

(ii) Every $i$-vertex with $2+k \leq i \leq 2 k+1$ has outdegree less than $i$.

\section{Proof.}

(i) Suppose, for a contradiction, that $v$ is a vertex contradicting the assertion and let $u$ be an outneighbour of $v$. Note that $u$ is a $\left(<\frac{3 k}{2}+2\right)$-vertex by definition of a discharging digraph.

Let $L$ be a 2-list-assignment of $G$. Let $S$ be the set of leaves of $A_{D}^{+}(u)$. By minimality, let $c$ be a $k$-improper $L$-colouring of $G-A_{D}^{+}(u)$.

$A_{D}^{+}(u)$ is a $(k, 2)$-quasi-arborescence: since it is dominated by $v$ in $D, u$ has outdegree less than $\frac{3 k}{2}+1$ and so at most $2 k-1$. Thus by Lemma 2 , we can extend $c$ to $G-v u$ so that $\operatorname{im}(u) \leq k-1$. Since the leaves have degree at most $3 \leq k$, the improperty of the leaves is at most $3 \leq k$. So we obtain a $k$-improper $L$-colouring of $G-u v$.

If $c(u) \neq c(v)$ or $i m_{G-u v}(v) \leq k-1$ then $c$ is a $k$-improper $L$-colouring of $G$. Otherwise all the $k+1$ neighbours of $v$ are coloured the same so recolouring $v$ with its other allowed colour yields a $k$-improper $L$-colouring of $G$.

Hence $G$ is $k$-improper 2-choosable which is a contradiction.

$\mathrm{RR} \mathrm{n}^{\circ} 5164$ 
(ii) Suppose, for a contradiction, that $v$ is an $i$-vertex contradicting the assertion.

Let $L$ be 2-list-assignment of $G$ and $c$ a $k$-improper $L$-colouring of $G-v$. There is a colour of $L(v)$, say $\alpha$, that is assigned to at most $k$ neighbours of $v$. Let $v_{1}, \ldots, v_{s}$ be these neighbours.

Let $G^{\prime}=G-\bigcup_{j=1}^{s} A_{D}^{+}\left(v_{j}\right)$. And set $c^{\prime}=c$ for every vertex of $G^{\prime}$ and every leaf of the $A_{D}^{+}\left(v_{j}\right)$. By Lemma 2 applied to each $A_{D}^{+}\left(v_{j}\right)$ (which are disjoint except possibly on their leaves), we can extend $c^{\prime}$ into a $k$-improper $L$-colouring of $G-v$ such that $i m\left(v_{j}\right) \leq k-1$ for $1 \leq j \leq s$. Now by definition of $c^{\prime}$, the only neighbours of $v$ that may be assigned $\alpha$ by $c^{\prime}$ are those of $\left\{v_{1}, \ldots, v_{s}\right\}$. Hence setting $c^{\prime}(v)=\alpha$, the $L$-colouring $c^{\prime}$ is $k$-improper.

Hence $G$ is $k$-improper 2-choosable which is a contradiction.

Analogously, one can prove the following lemma when $k=2$.

Lemma 5 Let $D$ be a discharging digraph of a (2,2)-minimal graph $G$.

(i) The outdegree of a 3-vertex is zero.

(ii) If $v$ is an $i$-vertex with $i \in\{4 ; 5\}$ then its outdegree is less than $i$.

Lemma 6 Let $D$ be a discharging of a $(1,2)$-minimal graph $G$. There is no 3-vertex with outdegree 3 in $D$.

Proof. Suppose, for a contradiction, that $v$ is a 3 -vertex with outdegree 3 . Let $u$ be an outneighbour of $v$. Let $Q_{1}=A_{D}^{+}(u), Q_{2}=A_{D-v u}^{+}(v), S$ be the set of leaves of $A_{D}^{+}(v)$ with indegree 1 in $A_{D}^{+}(v)$ and $S^{\prime}$ the set of leaves with indegree 2 in $A_{D}^{+}(v)$.

Let $L$ be a 2-list-assignment of $G$. By minimality of $G$, let $c$ be a 1-improper $L$-colouring of $G-A_{D}^{+}(v)$. Vertices not in $S$ have no neighbour in $G-A_{D}^{+}(v)$ and every vertex of $S$ has exactly one neighbour in $G-A_{D}^{+}(v)$. Extend $c$ to $S \cup S^{\prime}$ by assigning to each vertex of $S$ a colour of its list not assigned to its neighbour in $G-A_{D}^{+}(v)$ and any colour of its list to a vertex of $S^{\prime}$.

Now $Q_{1}$ and $Q_{2}$ satisfy either (i) or (ii) of Lemma 3. If one of them satisfies (ii), then possibly with recolouring of vertices of $S^{\prime}$ one can extend $c$ into a 1-improper $L$-colouring of $G-v u$ such that $c(v) \neq c(u)$. Hence $c$ is a 1-improper $L$-colouring of $G$.

If $Q_{1}$ and $Q_{2}$ satisfies both $(i)$, then possibly with recolouring of vertices of $S^{\prime}$ one can extend $c$ into a 1-improper $L$-colouring of $G-v u$ such that $i m(v)=i m(u)=0$. Hence $c$ is a 1-improper $L$-colouring of $G$.

So $G$ is 1-improper 2-choosable which is a contradiction.

Proof of Theorem 2. Let $G$ be a $(k, 2)$-minimal graph and $D$ a discharging digraph of $G$. We start with a charge $w(v)=d(v)$ on each vertex and we apply the following discharging rule: every vertex gives $\frac{k}{k+2}$ to each of its outneighbours.

Let us examine the new charge $w^{\prime}(v)$ of a vertex $v$ : 
- If $v$ is a 2-vertex, it has indegree 2 so its new charge is $w^{\prime}(v)=2+\frac{2 k}{k+2}=\frac{4 k+4}{k+2}$.

- If $v$ is a 3 -vertex and $k \geq 3$, it has indegree 3 so its new charge is $w^{\prime}(v)=3+3 \times \frac{k}{k+2}=$ $\frac{6 k+6}{k+2}>\frac{4 k+4}{k+2}$. If $v$ is a 3 -vertex and $k=2$ then it has outdegree 0 by Lemma 5 and indegree 0 by construction so $w^{\prime}(v)=3$.

- If $4 \leq d(v) \leq k+1,(k \geq 3)$, then by Lemma 4 (i), $v$ has outdegree zero so its charge is $d(v) \geq 4>\frac{4 k+4}{k+2}$.

- If $k+2 \leq d(v)<\frac{3 k}{2}+2$ then either $v$ has outdegree at most $d(v)-2$ and so its new charge is at least $d(v)-(d(v)-2) \times \frac{k}{k+2}=\frac{2 d(v)}{k+2}+\frac{2 k}{k+2} \geq 2+\frac{2 k}{k+2}=\frac{4 k+4}{k+2}$, or by Lemmas 4,5 and 6 , it has outdegree $d(v)-1$. In this case, by definition of a discharging digraph, $v$ has indegree 1 so its new charge is:

$$
d(v)-(d(v)-1) \times \frac{k}{k+2}+\frac{k}{k+2}=d(v)-(d(v)-2) \times \frac{k}{k+2} \geq \frac{4 k+4}{k+2} .
$$

- If $\frac{3 k}{2}+2 \leq d(v) \leq 2 k+1,(k \geq 2)$, then by Lemmas 4 and $5, v$ has outdegree at most $d(v)-1$. So $w^{\prime}(v) \geq d(v)-(d(v)-1) \times \frac{k}{k+2}=\frac{2 d(v)}{k+2}+\frac{k}{k+2} \geq \frac{3 k+4+k}{k+2}=\frac{4 k+4}{k+2}$.

- If $d(v) \geq 2 k+2$, then $w^{\prime}(v) \geq d(v)\left(1-\frac{k}{k+2}\right)=\frac{2 d(v)}{k+2} \geq \frac{4 k+4}{k+2}$.

Hence $\operatorname{Mad}(G) \geq \frac{1}{|V|} \sum_{v \in V} d(v)=\frac{1}{|V|} \sum_{v \in V} w^{\prime}(v) \geq \frac{4 k+4}{k+2}$.

Corollary 1 Let $G$ be a planar graph of girth $g$.

1. If $g \geq 8$ then $G$ is 1-improper 2-choosable, so $g_{1} \leq 8$.

2. If $g \geq 6$ then $G$ is 2-improper 2-choosable, so $g_{3} \leq g_{2} \leq 6$.

3. If $g \geq 5$ then $G$ is 4-improper 2 -choosable, so $g_{k} \leq 4$ for $k \geq 5$.

\subsection{Upper bound for $M(k, 2)$}

Let us fix $k \geq 1$. In this section, we shall construct a family of graphs $\left(G_{n}^{k}\right)_{n \geq 1}$ such that for all $n \geq 1$ :

- $G_{n}^{k}$ is not $k$-improper 2-colourable.

- $\operatorname{Mad}\left(G_{n}^{k}\right)=\frac{2 n\left(4 k^{2}+6 k+4\right)+4 k^{2}+6 k+2}{2 n\left(k^{2}+2 k+2\right)+(k+1)^{2}}$.

$\mathrm{RR} \mathrm{n}^{\circ} 5164$ 
Hence we will deduce:

Theorem 3 For all $k \geq 1, M(k, 2) \leq \frac{4 k^{2}+6 k+4}{k^{2}+2 k+2}=4-\frac{2 k+4}{k^{2}+2 k+2}$.

We denote by $H_{k}$ the graph composed of two adjacent vertices $u$ and $v$ also connected by $k+1$ disjoint paths of length 2. Take $k$ copies of $H_{k}$ and create the graph $F_{k}$ by identifying the vertex $v$ of each copy. Note that $F_{k}$ has one vertex of degree $k(k+2), k$ vertices of degree $k+2$ and $k(k+1)$ vertices of degree 2 . Now we take $2 n+1$ copies of $F_{k}$ and we join the vertices $v$ of each copy creating a cycle of size $2 n+1$. At last we make a subdivision of all the edges of the cycle but one so as to obtain the graph $G_{n}^{k}$.

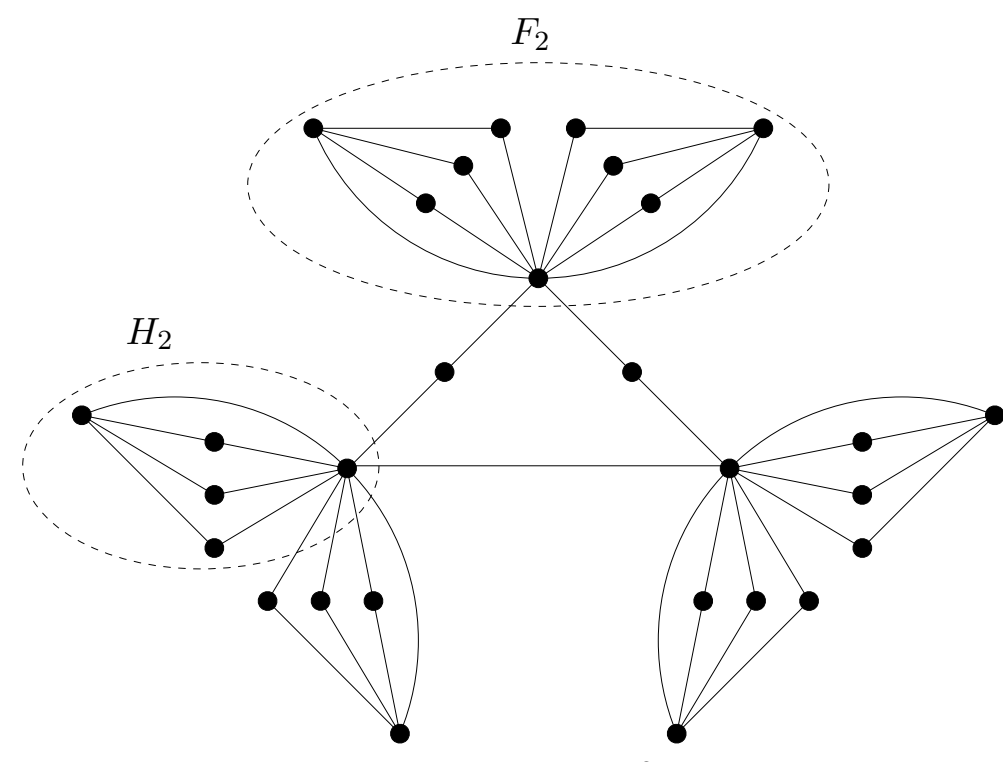

The graph $G_{1}^{2}$

Lemma $7 G_{n}^{k}$ is not k-improper 2-colourable.

Proof. First remark that in any $k$-improper 2 colouring of $H_{k}, v$ has improperty at least 1. Indeed $v$ is a $(k+2)$ vertex in $H_{k}$, so if it has improperty zero then its $k+2$ neighbours are coloured the same, but this is impossible since $u$ is a neighbour of $v$ adjacent to the $k+1$ remaining neighbours. Hence in any $k$-improper colouring of $F_{k}, v$ has improperty $k$. So in order to colour the whole graph, we must properly colour the subdivided cycle with 2 colours, which is impossible.

Lemma 8 The maximum average degree of $G_{n}^{k}$ is $M_{n}^{k}=\frac{(2 n+1)\left(4 k^{2}+6 k+4\right)-2}{(2 n+1)\left(k^{2}+2 k+2\right)-1}$. 
Proof. As it is easily seen, the maximum average degree of $G$ is its average degree, which is:

$$
\frac{(2 n+1)[(1 \times k(k+2)+2)+(k \times(k+2))+(k(k+1) \times 2)]+(2 n) \times 2}{(2 n+1)(1+k+k(k+1))+2 n}=M_{n}^{k} .
$$

\section{Improper l-choosability, $l \geq 2$}

\subsection{Lower bound for $M(k, l)$}

In this subsection, we shall prove the following theorem:

Theorem 4 For all $l \geq 2$ and all $k \geq 0$, all graphs of maximum average degree less than $\frac{l(l+2 k)}{l+k}$ are $k$-improper l-choosable.

The result of the theorem is trivial if $k=0$ since a graph of maximum average degree less than $l$ is $(l-1)$-degenerate (i.e. each of its subgraph has a vertex of degree at most $l-1$ ). Hence it is $l$-choosable. For bigger values of $k$, we will need some preliminary results.

Definition 5 A graph is said to be $(k, l)$-minimal if it is not $k$-improper $l$-choosable but every of its proper subgraph is.

Lemma 9 Let $G$ be a graph, $L$ a list-assignment and $c$ an $L$-colouring. If a vertex $v$ has improperty at least $d(v)-|L(v)|+2$ under $c$, then there exists an L-colouring $c^{\prime}$ of $G$ such that $c^{\prime}(u)=c(u)$ if $u \neq v$ and $\operatorname{im}_{c^{\prime}}(v)=0$.

Proof. Let $c(v)=\alpha$. Then $v$ has at most $d(v)-(d(v)-|L(v)|+2)=|L(v)|-2$ neighbours that are not coloured with $\alpha$. Hence there exists a colour $\beta \in L(v)$ that does not colour any neighbour of $v$. So setting $c(v)=\beta$ we obtain the desired colouring.

We now prove a generalization of Lemma 1.

Lemma 10 Let $k \geq 1$ and let $G$ be a $(k, l)$-minimal graph. Then:

(i) $\delta \geq l$.

(ii) Two $(\leq l+k-1)$-vertices are not adjacent.

\section{Proof.}

$\mathrm{RR} \quad \mathrm{n}^{\circ} 5164$ 
(i) Let $L$ be an $l$-list-assignment and suppose $v$ is a $(\leq l-1)$-vertex. By minimality let $c$ be a $k$-improper $L$-colouring of $G-v$. As $v$ has at most $l-1$ neighbours in $G$, there exists a colour, say $\alpha$, that is not assigned to any neighbour of $v$. Hence colouring $v$ with $\alpha$ yields a $k$-improper $L$-colouring of $G$.

Hence $G$ is $k$-improper $l$-choosable, a contradiction.

(ii) Let $L$ be an $l$-list-assignment and suppose, for a contradiction, that $u$ and $v$ are two neighbours of degree at most $l+k-1$. By minimality, let $c$ be a $k$-improper $L$-colouring of $G-\{u v\}$. Then $c$ is an $L$-colouring of $G$ such that each vertex has improperty at most $k$, except possibly $u$ and $v$ which may have improperty $k+1$. But in this case we use Lemma 9 to recolour these vertices and obtain a $k$-improper $L$-colouring of $G$. Hence $G$ is $k$-improper $l$-choosable, a contradiction.

Definition 6 Let $G$ be a $(k, l)$-minimal graph. We partially orient $G$ using the following process:

1. Orient each edge $u v$ where $v$ is a $(\leq l+k-1)$-vertex from $u$ to $v$.

2. While there is an $i$-vertex $v$ with $l+k \leq i<l+k+\frac{k}{l}$ having outdegree exactly $i-l+1$ and indegree 0 , we orient one of its unoriented incident edges $u v$ from $u$ to $v$.

The digraph $D$ induced by the oriented edges is called a discharging digraph of $G$.

The following remark follows from the definition of a discharging digraph.

\section{Remark 1}

- Only vertices of degree less than $l+k+\frac{k}{l}$ can have indegree more than zero.

- For $i \leq l+k-1$, every $i$-vertex has indegree exactly $i$ in $D$.

Definition 7 A quasi-arborescence rooted at $u$ is a $(k, l)$-quasi-arborescence if and only if:

- Every vertex has outdegree at most $\max \{2,2 k-1\}$.

- Every leaf has indegree at most $l+k-1$

Now we generalize Lemmas 2 and 3 .

Lemma 11 Let $k \geq 2$ and let $Q$ be a $(k, l)$-quasi-arborescence rooted at $u$. Let $L$ be a list-assignment of $Q$ such that $|L(v)| \geq \max \left\{1, d_{Q}(v)-k+1\right\}$ if $v$ is a leaf and $|L(v)| \geq 2$ otherwise. We denote by $S$ the set of leaves that have indegree at least $k+1$ in $Q$ (and hence a colour-list of size at least 2). Any L-colouring of the leaves extends in an L-colouring of $Q$ such that: 
- $i m(u) \leq k-1$.

- $\forall v \notin S, i m(v) \leq k$.

Furthermore, possibly by recolouring some vertices of $S$, this $L$-colouring of $G$ can be made k-improper.

Proof. By induction on the number of vertices of $Q$, the result being trivially true if $|V(Q)|=1$.

Suppose now that $|V(Q)|>1$ and the result holds for smaller $(k, l)$-quasi-arborescences. Let $v_{1}, \ldots, v_{s}$ be the outneighbours of $u$ in $Q$. Note that $Q-u$ is the union of $s(k, l)$-quasiarborescences $Q_{i}$ rooted at $v_{i}, 1 \leq i \leq s$, that are disjoint except possibly on their leaves. We start by $L$-colouring all the leaves of $Q$.

By induction we extend this colouring to an $L$-colouring of each of the $Q_{i}$ such that $i m\left(v_{i}\right) \leq k-1$. Note that $i m_{Q}(x)=i m_{Q_{i}}(x) \leq k$ for every vertex of $Q_{i}$ which is not a leaf and $i m_{Q}(x) \leq k$ for each leaf not in $S$. One of the two colours of $L(u)$, say $\alpha$, is assigned to at most $k-1$ neighbours of $u$ since $\operatorname{deg}(u) \leq 2 k-1$. Hence setting $c(u)=\alpha$, we obtain the first desired colouring.

Now, we can recolour each leaf $f$ of $S$ with improperty at least $k+1$ using Lemma 9 since $d_{Q}(f)-|L(f)|+2 \leq d_{Q}(f)-d_{Q}(f)+k-1+2=k+1$. This concludes the proof.

The above result cannot be extended for $k=1$. However one can prove the following:

Lemma 12 Let $Q$ be a $(1, l)$-quasi-arborescence rooted at $u, L$ be a list-assignment of $Q$ such that $|L(v)| \geq 2$ if $v$ is not a leaf, and $|L(v)| \geq d_{Q}(v)$ otherwise. We denote by $S$ the set of leaves with indegree at least 2. Let $c$ be an L-colouring of the leaves. One of the followings holds:

(i) $c$ can be extended in an $L$-colouring of $Q$ such that im $(u)=0$ and $\operatorname{im}(v) \leq 1$ if $v \notin S$;

(ii) $c$ can be extended in two different $L$-colourings of $Q c_{1}$ and $c_{2}$ such that $c_{1}(v)=c_{2}(v)$ if $v \neq u$ and $\mathrm{im}^{c_{i}}(v) \leq 1$ if $v \notin S$.

Furthermore, possibly by recolouring vertices of $S$, all these L-colourings can be made 1improper.

Moreover, if $|L(u)| \geq 3$ then (i) holds.

Proof. By induction on the number of vertices, the result being obvious if $|V(Q)|=1$.

$Q-u$ is the union of two $(1, l)$-quasi-arborescences $Q_{1}$ and $Q_{2}$ rooted at $v_{1}$ and $v_{2}$ respectively. They are disjoint except possibly on their leaves. Let $c$ be an $L$-colouring of the leaves of $Q$. By induction we extend $c$ to $Q_{1}$ and $Q_{2}$. Note that for each vertex $v$ of $Q-S i m_{Q}(v)=i m_{Q_{i}}(v) \leq 1$.

If at least one of the $Q_{i}$ satisfies (ii), or if $|L(u)| \geq 3$, we can suppose that $\left\{c\left(v_{1}\right), c\left(v_{2}\right)\right\} \neq$ $L(u)$ and hence we extend $c$ into an $L$-colouring of $Q$ fulfilling $(i)$.

$\mathrm{RR} \mathrm{n}^{\circ} 5164$ 
If both $Q_{1}$ and $Q_{2}$ satisfy $(i)$, then either $c\left(v_{1}\right)=c\left(v_{2}\right)$ and hence setting $c(u) \in L(u) \backslash$ $\left\{c\left(v_{1}\right)\right\}$ yields an $L$-colouring of $Q$ that satisfies $(i)$; or colouring $u$ with two colours of its list gives the two desired colourings of $(i i)$.

Now we can recolour with improperty zero each leaf $f \in S$ that has improperty at least 2 in $Q$ using Lemma 9, since $d_{Q}(f)-|L(f)|+2 \leq 2$. This concludes the proof.

Using these results, we can say more about the structure of a discharging digraph. The following lemma generalizes Proposition 1.

Lemma 13 Let $D$ be a discharging digraph of a $(k, l)$-minimal graph $G$.

(i) Every vertex $u$ with $l+k \leq d(u) \leq l+2 k-1$ has outdegree at most $d(u)-l+1$. In particular, $D$ is acyclic.

(ii) For every vertex $u, A_{D}^{+}(u)$ is a $(k, l)$-quasi-arborescence. In particular, the indegree of the leaves in $A_{D}^{+}(u)$ is at most $l+k-1$.

Proof. (ii) follows easily from (i). So, let us prove (i).

Let $L$ be an $l$-list-assignment of $G$. First, $D$ has no 2-circuit since two $(\leq l+k-1)$ vertices are not adjacent by Lemma 10 . Note also that in order to create a circuit in $D$, it is necessary to create a vertex $u$ of outdegree at least $d(u)-l+2$. Now suppose, for a contradiction, that $D$ contains a vertex $u$ of outdegree at least $d(u)-l+2$ and let $D^{\prime}$ be the digraph obtained just after having created the first such vertex $u$. Let $u \rightarrow v$ be the last edge that is oriented in $D^{\prime} . u$ has $d(u)-l+2$ outneighbours (including $v$ ) while $v$ has $d(v)-l+1$ outneighbours. We distinguish two cases depending whether the orientation of $u v$ creates a circuit (which is necessary the first), or not.

First Case: the orientation of $u v$ creates a circuit $C$. Let $w$ be the inneighbour of $u$ in $C$. We define $Q_{1}=A_{D^{\prime}-w u}^{+}(v), Q_{2}=A_{D^{\prime}-u v}^{+}(u)$ and $Q=Q_{1} \cup Q_{2}$. Note that $Q_{1}$ and $Q_{2}$ are $(k, l)$-quasi-arborescences which are disjoint, except possibly on some leaves. In particular the outdegree in $D^{\prime}$ of every internal vertex $x$ of $Q$ is at most $d_{G}(x)-l+1$. More precisely every internal vertex $x \neq w$ satisfies $d_{D^{\prime}}^{+}(x)=d_{G}(x)-l+1$ while $d_{D^{\prime}}^{+}(w)=d_{G}(w)-l$ and for all every vertex $v d_{D^{\prime}}^{-}(x)=1$. Recall that $d_{Q}(w)=d^{+}(w)+d^{-}(w)$. Let $F$ be the set of leaves in $Q, S$ the set of leaves that have indegree at least $k+1$ in $Q$ and $\bar{S}=F \backslash S$. We define

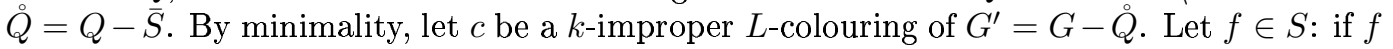
has improperty at least $k-d_{Q}(f)+1$, then using Lemma 9 we recolour it with improperty 0 since $d_{G^{\prime}}(f)-|L(f)|+2=d_{G}(f)-d_{Q}(f)-l+2 \leq l+k-1-d_{Q}(f)-l+2=k-d_{Q}(f)+1$. Now, let $L_{1}$ be the following list-assignment of $Q_{1}$ :

$L_{1}(x)=L(x) \backslash\left\{\alpha \mid \exists z \in N_{G-Q_{1}}(x), c(z)=\alpha\right\}$ if $x \notin \bar{S}$, and $L_{1}(x)=\{c(x)\}$ otherwise. Note that if $x \neq w$ is an internal vertex then:

$$
\left|L_{1}(x)\right| \geq l-\left(d_{G}(x)-d_{Q_{1}}(x)\right)=l-d_{G}(x)+d_{G}(x)-l+1+1=2
$$

and since $d^{+}(w)=d_{G}(w)-l$ but $u$ is yet uncoloured:

$$
\left|L_{1}(w)\right| \geq l-\left(d_{G}(w)-d_{Q_{1}}(w)\right)+1=l-d_{G}(w)+d_{G}(w)-l+1+1=2 .
$$


For the root $v, d^{-}(v)=0$ but $u$ is uncoloured yet so:

$$
\left|L_{1}(v)\right| \geq l-\left(d_{G}(v)-d_{Q_{1}}(v)\right)+1=l-d_{G}(v)+d_{G}(v)-l+1+1=2,
$$

and for a leaf $f \in S$ :

$$
\left|L_{1}(f)\right| \geq l-d_{G}(f)+d_{Q}(f) \geq l-(l+k-1)+d_{Q}(f)=d_{Q}(f)-k+1 .
$$

Thus we may apply Lemmas 11 and 12 . To do so, we $L_{1}$-colour all the leaves in $Q$.

Suppose first $k \geq 2$. By Lemma 11, we obtain an $L_{1}$-colouring $c_{1}$ of $Q_{1}$ such that $i m_{Q_{1}}^{c_{1}}(v) \leq k-1$. Note that $c_{1}$ extends $c$ into an $L$-colouring of $G-Q_{2}$ such that each vertex has improperty at most $k$ except possibly some vertices of $S$. Furthermore, $i m_{G-Q_{2}}(v) \leq k-$ 1. We define a list-assignment $L_{2}$ of $Q_{2}$ by $L_{2}(u)=L(u) \backslash\left\{\alpha \mid \exists z \neq v \in N_{G-Q_{2}}(u), c(z)=\right.$ $\alpha)\}, L_{2}(x)=\{c(x)\}$ if $x$ is a leaf and $L_{2}(x)=L(x) \backslash\left\{\alpha \mid \exists z \in N_{G-Q_{2}}(x), c(z)=\alpha\right\}$ otherwise. Note that we have $\left|L_{2}(u)\right| \geq 2$. We now apply Lemma 11 so as to get an $L_{2}$-colouring of $Q_{2}$ and hence an $L$-colouring of $G$. Every vertex not in $S \cup\{u, v\}$ has improperty at most $k$. If $x \in\{u, v\}$ then: $\operatorname{im}_{G}(x) \leq i m_{G-Q_{i}}(x)+1 \leq k-1+1=k$ since there cannot be in $L_{2}(u)$ the colour of a neighbour of $u$ in $G-\left(Q_{2}-v\right)$. If $f \in S$ has improperty at least $k+1$, then we recolour it with improperty 0 using Lemma 9 since $d_{G}(f)-|L(f)|+2 \leq l+k-1-l+2=k+1$. Thus we obtain a $k$-improper $L$-colouring of $G$.

Suppose now $k=1$. Applying Lemma 12 , we obtain an $L_{1}$-colouring of $G-Q_{2}$ such that every vertex not in $S$ has improperty at most 1, and either $v$ has improperty $0(i)$, or it has improperty 1 and we can indifferently colour it with two colours of its list (ii). Note that if $v$ has one neighbour distinct from $u$ which is an internal vertex in $Q_{2}$ then $\left|L_{1}(v)\right| \geq 3$ so we may suppose that $v$ fulfils $(i)$. Defining $L_{2}$ as before, we can apply Lemma 12 to $Q_{2}$ so as to obtain an $L_{2}$-colouring of $Q_{2}$ and hence an $L$-colouring of $G$ such that $u$ fulfils $(i)$ or $(i i)$. Now, every vertex not in $S \cup\{u, v\}$ has improperty at most 1 . If $v$ satisfies $(i)$, then either $u$ also satisfies $(i)$ or $u$ satisfies $(i i)$ but in this case we may suppose $u$ and $v$ are coloured differently so in all cases they have improperty at most 1 in $G$. If $v$ satisfies (ii), then the only neighbour of $v$ in $Q_{2}$ is $u$. Hence we may safely suppose that $u$ and $v$ are coloured differently, so they have improperty at most 1 in $G$.

Finally, we can recolour each leaf of $S$ that has improperty at least 2 by using Lemma 9 and thus we obtain a 1-improper $L$-colouring of $G$.

Hence $G$ is $k$-improper $l$-choosable, a contradiction.

Second Case: there is no circuit in $D^{\prime}$. Then $Q=A_{D^{\prime}}^{+}(u)$ is a quasi-arborescence. Moreover each internal vertex $v$ has outdegree at most (and hence exactly) $d(v)-l+1$. Let $v_{1}, \ldots, v_{s}$ be the outneighbours of $u$, we define $Q_{j}=A_{D^{\prime}}^{+}\left(v_{j}\right), 1 \leq j \leq s$. The $Q_{i}$ are $(k, l)$-quasi-arborescences that are disjoint except possibly on their leaves. Let $F$ be the set of leaves in $Q, S$ the set of leaves with indegree at least $k+1$ in $Q$ and $\bar{S}=F \backslash S$. We define $Q=Q-\bar{S}$. Let $L$ be an $l$-list-assignment of $G$. By minimality, let $c$ be a $k$ improper $L$-colouring of $G^{\prime}=G-\grave{Q}$. Let $f$ be a leaf in $\bar{S}$. If $f$ has improperty at least 
$k-d_{Q}(f)+1$, we recolour it with improperty 0 using Lemma 9 since: $d_{G^{\prime}}(f)-|L(f)|+2 \leq$ $d_{G}(f)-d_{Q}(f)-l+2 \leq l+k-1-d_{Q}(f)-l+2=k-d_{Q}(f)+1$.

For each vertex $v \in Q$, we define $L^{\prime}(v)=L(v) \backslash\left\{\alpha \mid \exists w \in N_{G}(v), c(w)=\alpha\right\}$ if $v \notin \bar{S}$ and $L^{\prime}(v)=\{c(v)\}$ otherwise. Note that for an internal vertex $v$ :

$$
\left|L^{\prime}(v)\right| \geq l-\left(d_{G}(v)-d_{Q}(v)\right)=l-d_{G}(v)+d_{G}(v)-l+1+1=2 .
$$

For a leaf $f \in S$ :

$$
\left|L^{\prime}(f)\right| \geq l-d_{G}(f)+d_{Q}(f) \geq l-(l+k-1)+d_{Q}(f)=d_{Q}(f)-k+1 .
$$

Suppose first $k \geq 2$. We $L^{\prime}$-colour all the leaves, use Lemma 11 so as to extend it into an $L^{\prime}$-colouring of each of the $Q_{i}$, and possibly with recolouring some leaves in $S$ we get a $k$-improper $L$-colouring of $G-u$ such that $i m\left(v_{j}\right) \leq k-1,1 \leq j \leq s$.

Now $\left|L^{\prime}(u)\right| \geq|L(u)|-d(u)+d_{D^{\prime}}^{+}(u)=l-d(u)+d(u)-l+2 \geq 2$. And $u$ has $d^{+}(u)=d(u)-l+2 \leq 2 k+1$ outneighbours in $D^{\prime}$. Thus there is a colour of $L^{\prime}(u)$, say $\alpha$, that is assigned to at most $k$ outneighbours of $u$. Thus setting $c(u)=\alpha$ yields a $k$-improper $L$-colouring of $G$ by definition of $L^{\prime}$.

Suppose now $k=1$. We $L^{\prime}$-colour all the leaves, use Lemma 12 so as to extend it in an $L^{\prime}$-colouring of each of the $Q_{i}$, and possibly with recolouring some leaves in $S$ we get a 1-improper $L$-colouring of $G-u$ such that for each $v_{j}$ either $i m\left(v_{j}\right)=0$ or $v_{j}$ can safely be recoloured with another colour of $L^{\prime}\left(v_{j}\right)$.

The same calculation as above shows there exists a colour of $L^{\prime}(u)$, say $\alpha$, that is assigned to at most 1 neighbour of $u$, say $v_{i}$. We set $c(u)=\alpha$. If $v_{i}$ satisfies the first condition, we have a 1-improper $L$-colouring of $G$. If $v_{i}$ satisfies the second condition then we may suppose that $c(u) \neq c(v)$ and thus we also have a 1-improper $L$-colouring of $G$.

Hence $G$ is $k$-improper $L$-choosable, a contradiction

Proof of Theorem 4. Let $G$ be a $(k, l)$-minimal graph and $D$ a discharging digraph of $G$. We start with a charge $w(v)=d(v)$ on each vertex and we apply the following discharging rule: every vertex gives $\frac{k}{l+k}$ to each of its outneighbours.

Let us examine the new charge $w^{\prime}(v)$ of a vertex $v$ :

- If $d(v) \leq l+k-1$ it has indegree $d(v)$ so its new charge is $w^{\prime}(v)=d(v)+\frac{d(v) k}{l+k} \geq l+\frac{l k}{l+k}$.

- If $l+k \leq d(v)<l+k+\frac{k}{l}$ then either $v$ has outdegree at most $d(v)-l$ and so its new charge is at least $d(v)-(d(v)-l) \times \frac{k}{l+k}=\frac{l d(v)}{l+k}+\frac{l k}{l+k} \geq l+\frac{l k}{l+k}$, or by Lemma 13 , it has outdegree $d(v)-l+1$. In this case, by definition of a discharging digraph, $v$ has indegree 1 so its new charge is:

$$
w^{\prime}(v)=d(v)-(d(v)-l+1) \times \frac{k}{l+k}+\frac{k}{l+k}=d(v)-(d(v)-l) \times \frac{k}{l+k} \geq l+\frac{k}{l+k} .
$$


- If $l+k+\frac{k}{l} \leq d(v) \leq l+2 k-1$, then by Lemma $13, v$ has outdegree at most $d(v)-l+1$. So $w^{\prime}(v) \geq d(v)-(d(v)-l+1) \times \frac{k}{l+k}=\frac{l d(v)}{l+k}+\frac{k l-k}{l+k} \geq \frac{l^{2}+2 k l}{l+k}=l+\frac{k l}{l+k}$.

- If $d(v) \geq l+2 k$, then $w^{\prime}(v) \geq d(v)\left(1-\frac{k}{l+k}\right)=\frac{l d(v)}{l+k} \geq \frac{l^{2}+2 k l}{l+k}=l+\frac{k l}{l+k}$.

Hence $\operatorname{Mad}(G) \geq \frac{1}{|V|} \sum_{v \in V} d(v)=\frac{1}{|V|} \sum_{v \in V} w^{\prime}(v) \geq l+\frac{k l}{l+k}$.

\section{$3.2 \quad$ Upper bound for $M(k, l)$}

In this section we shall construct for all $l \geq 2$ and all $k \geq 1$, a graph $G_{l}^{k}$ which is not $k$ improper $l$-colourable. So its maximum average degree will give an upper bound for $M(k, l)$. To construct $G_{2}^{k}$, take $k+1$ copies of $H_{k}$ (defined in Subsection 2.2) and identify their vertex $v$. We define $G_{l}^{k}, l \geq 3$, inductively. First we create the graph $M_{l}^{k}$ by taking $k$ copies of $G_{l-1}^{k}$ and adding a vertex $w$ which we join to every other vertices. Then we take $l-1$ copies $M^{1}, \ldots, M^{l-1}$ of $M_{l}^{k}$ and we join all the vertices $w_{1}, \ldots, w_{l-1}$ (so that they form a complete graph of size $l-1$ ). Now, we add $k+2$ vertices $z_{0}, z_{1}, \ldots, z_{k+1}$ each joined to each of the $w_{i}, 1 \leq i \leq l-1$. Last we add the edges $z_{0} z_{i}$ for $1 \leq i \leq k+1$.

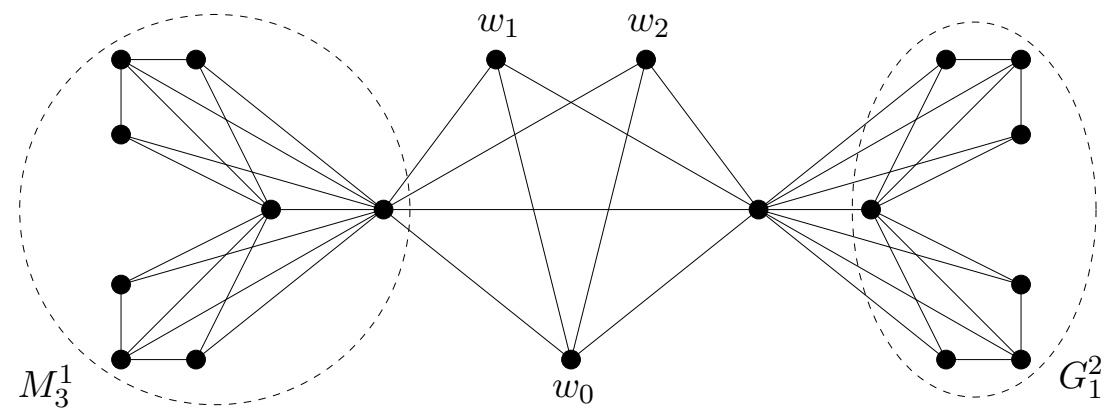

The graph $G_{3}^{1}$

Lemma 14 For all $l \geq 2$ and all $k \geq 1$, the graph $G_{l}^{k}$ is not $k$-improper l-colourable.

Proof. The result is clear for $G_{2}^{k}$. Suppose the result is true for $l-1 \geq 2$ and let us prove it for $G_{l}^{k}$. First note that in any $k$-improper $l$-colouring of $M^{i}$, the vertex $w_{i}$ has improperty $k$. Indeed, $w_{i}$ has a neighbour of its colour in each copy of $G_{l-1}^{k}$ since otherwise $G_{l-1}^{k}$ would be $k$-improper $(l-1)$-colourable. Hence each of the $w_{i}, 1 \leq i \leq l-1$, cannot have any neighbour of its colour in $G_{l}^{k}-M^{i}$. In particular, as the subgraph induced by $w_{1}, \ldots w_{l-1}$ 
is complete, all the $z_{i}, 0 \leq i \leq k+1$, must be coloured the same. But then $w_{0}$ must have improperty $k+1$.

Lemma 15 The number of vertices of $G_{l}^{k}$ is:

$$
n_{l}^{k}=2 l+(l+1) k+\sum_{i=2}^{l} \frac{(l-1) !}{(l-i) !} k^{i} .
$$

In particular, it is a polynom in $k$ of degree $l$ and dominant coefficient $(l-1)$ !.

Proof. $n_{l}^{k}$ satisfies: $n_{2}^{k}=k^{2}+3 k+3$ and $\forall l \geq 3, n_{l}^{k}=\left(k \times n_{l-1}^{k}+1\right) \times(l-1)+k+2$.

Let $s_{l}^{k}$ denotes the sum of the degrees of the vertices in $G_{l}^{k}$. We have the following result:

Lemma $16 s_{l}^{k}$ is a polynom in $k$ of degree $l$ whose dominant coefficient is $2 l$ !.

Proof. $s_{l}^{k}$ satisfies: $s_{2}^{k}=4 k^{2}+10 k+6$ and $s_{l}^{k}=(l-1)\left(k \times s_{l-1}^{k}+2 k \times n_{l-1}^{k}+l+k\right)+(l+1) k+2 l$ if $l \geq 3$. Hence it is a polynom in $k$ of degree $l$. Furthermore, denoting by $c_{l}^{k}$ its dominant coefficient, we have: $c_{2}^{k}=4$ and $\forall l \geq 3, c_{l}^{k}=(l-1) \times c_{l-1}^{k}+2 k \times(l-1)$ !. Thus $c_{l}^{k}=2 l !$.

Proposition $2 \lim _{k \rightarrow \infty} \operatorname{Mad}\left(G_{l}^{k}\right)=2 l$.

Proof. It is clear that the maximum average degree of $G_{l}^{k}$ is its average degree. Then by Lemmas 15 and 16, we have:

$$
\lim _{k \rightarrow \infty} \operatorname{Mad}\left(G_{l}^{k}\right)=2 \frac{l !}{(l-1) !}=2 l .
$$

Corollary 2 For any fixed $l, \lim _{k \rightarrow+\infty} M(k, l)=2 l$.

Proof. It follows from Theorem 4 and Proposition 2.

\section{References}

[1] K. Appel and W. Haken, Every planar map is four colourable, Part I: Discharging, Ilinois J. Math. 21 (1977), 429-490.

[2] K. Appel, W. Haken and J. Koch Every planar map is four colourable, Part II: Reducibility, Illinois J. Math. 21 (1977), 491-567.

INRIA 
[3] N. Eaton and T. Hull, Defective list colorings of planar graphs, Bull. Inst. Combin. Appl. 25 (1999), 79-87.

[4] H. Grötzsch, Ein Dreifarbensatz für dreikreisfreie Netze auf der Kugel, Wiss. Z. Martin Luther Univ. Halle-Wittenberg, Math.-nat 8 (1959), 109-120.

[5] K.-W. Lih, Z. Song, W. Wang and K. Zhang, A Note on List Improper Coloring Planar Graphs, Appl. Math. Let. 14 (2001), 269-273.

[6] R. Škrekovski, List improper colouring of planar graphs, Comb. Prob. Comp. 8 (1999), 293-299.

[7] R. Škrekovski, A Grötzsch-type theorem for list colourings with impropriety one, Comb. Prob. Comp. 8 (1999), 493-507.

[8] R. Škrekovski, List improper colorings of planar graphs with prescribed girth, Discrete Math. 214 (2000), 221-233.

[9] C. Thomassen, Every planar graph is 5-choosable, J. Comb. Theory B 62 (1994), $180-181$.

[10] C. Thomassen, 3-list-coloring planar graph of girth 5, J. Comb. Theory B 64 (1995), 101-107.

[11] C. Thomassen, A short list color proof of Grötzsch's theorem, J. Comb. Theory B $\mathbf{8 8}$ (2003), 189-192.

[12] M. Voigt, List colorings of planar graphs, Discrete Math. 120 (1993), 215-219.

[13] M. Voigt, A not 3-choosable graph without 3-cycles, Discrete Math. 146 (1995), 325328.

$\mathrm{RR} \mathrm{n}^{\circ} 5164$ 


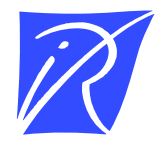

\section{Unité de recherche INRIA Sophia Antipolis 2004, route des Lucioles - BP 93 - 06902 Sophia Antipolis Cedex (France)}

Unité de recherche INRIA Futurs : Parc Club Orsay Université - ZAC des Vignes 4, rue Jacques Monod - 91893 ORSAY Cedex (France)

Unité de recherche INRIA Lorraine : LORIA, Technopôle de Nancy-Brabois - Campus scientifique 615, rue du Jardin Botanique - BP 101 - 54602 Villers-lès-Nancy Cedex (France)

Unité de recherche INRIA Rennes : IRISA, Campus universitaire de Beaulieu - 35042 Rennes Cedex (France)

Unité de recherche INRIA Rhône-Alpes : 655, avenue de l'Europe - 38334 Montbonnot Saint-Ismier (France)

Unité de recherche INRIA Rocquencourt : Domaine de Voluceau - Rocquencourt - BP 105 - 78153 Le Chesnay Cedex (France)

INRIA - Domaine de Voluceau - Rocquencourt, BP 105 - 78153 Le Chesnay Cedex (France)

http://www.inria.fr

ISSN 0249-6399 